This is the peer reviewed version of the following article:Navarro-Vázquez, A., Santamaría-Fernández, R., \& Sardina, F. (2018). MSpin-JCoupling. A modular program for prediction of scalar couplings and fast implementation of Karplus relationships. Magnetic Resonance In Chemistry, 56(6), 505-512, which has been published in final form at https://doi.org/10.1002/mrc.4667.

This article may be used for non-commercial purposes in accordance with Wiley Terms and Conditions for Use of Self-Archived Versions 


\title{
MSpin-JCoupling. A modular program for prediction of scalar couplings and fast implementation of Karplus relationships
}

\author{
Armando Navarro-Vázquez, ${ }^{* \dagger}$ Raquel Santamaría-Fernández, Francisco Javier \\ Sardina López.
}

Departamento de Química Orgánica and Centro Singular de Investigación en Química Biolóxica e Materiais Moleculares (CIQUS), Universidade de Santiago de Compostela, 15782 Santiago de Compostela, Spain

\begin{abstract}
MSpin-JCoupling is a modular program for the prediction of scalar couplings using a large variety of Karplus relationships. The program was specially designed for small molecule analysis and can be run in graphical or command-line mode. The architecture of the program is highly modular and new equations can be rapidly implemented, through a complete $\mathrm{C}++$ programming interface, and deployed as run-time loadable plugins.
\end{abstract}

Keywords: Scalar Couplings, Karplus Equation, Software, Open Source.

\footnotetext{
† Correspondence to: Armando Navarro-Vázquez, Actual address: Departamento de Química Fundamental, Universidade Federal de Pernambuco, PE50740-560, armando.navarro@ufpe.br
} 


\section{Introduction}

Scalar couplings ${ }^{[1-3]}$ have been, along with NOE effects, ${ }^{[4,5]}$ the two major and most intensively used NMR tools for the configurational and conformational analysis of small organic molecules. ${ }^{[6]}$ Their power reside on the fact that three-bond couplings $\left(3 \mathrm{~J}_{\mathrm{XY}}\right)$ have a strong dependence on the dihedral angle between the coupled nuclei. ${ }^{[7]} \mathrm{A}$ fair approximation to this dependence is the well-known Karplus equation, a three-term cosine Fourier series. ${ }^{[8]}$

$$
{ }^{3} J=A \cos 2 \phi+B \cos \phi+C
$$

From valence bond computation of the Fermi Contact term ${ }^{[7,8]}$ Karplus derived values of $4.5,-0.5$ and $4.22 \mathrm{~Hz}$ for $A, B$ and $C$ respectively but they can be adjusted to better fit experimental couplings in molecules of known geometry. In many cases the equation is recast as

$$
{ }^{3} J=A^{\prime} \cos ^{2} \phi+B^{\prime} \cos \phi+C^{\prime}
$$

where $A^{\prime}=2 A, C^{\prime}=C-A$ and $B^{\prime}=B$. The most usual set of coefficients employed in the Karplus equation are however those proposed by Altona and coworkers ${ }^{[9]}$ where $A^{\prime}=7.76, B^{\prime}=-1.10$ and $C^{\prime}=1.40$.

Different equations have been developed from the seminal work originally proposed by Karplus. Apart from the dihedral angle, the value of the three-bond $\left(3 J_{x y}\right) J$-coupling constants also depends on i) the electronegativity of the substituents attached to the spin system, ii) the bond lengths and iii) the valence angle as a result of ring tension and carbons hybridization. ${ }^{[10]}$ Many equations that take into account the influence of structural parameters beyond the dihedral angle were proposed for the prediction of $33_{\mathrm{HH}}$ couplings. Particularly, It was early recognized that the presence of electronegative substituents on the carbon atoms would generally lead to a decrease on the observed couplings. Hence, basic modifications to the Karplus equation, as the Durette and Horton equation, ${ }^{[11]}$ ( Eq. (0.0) )were proposed.

$$
{ }^{3} J=(5.6 \cos 2 \phi-1.0 \cos \phi+7.8)(1-1.0 \Delta \chi)
$$


where $\Delta \chi$ is the sum of the difference of electronegativies between the $\alpha$ substituents and that of hydrogen. However, it was also early noted that the effect of electronegative substituents depended also on their relative position respect to the coupled protons, leading therefore to a phase displacement on the $J(\phi)$ curve. For a single $X$ substituent this asymmetry can be taken into account by introducing extra sine terms, dependent on the nature of the substituent, in the Fourier expansion $^{[12]}$

$$
{ }^{3} J_{X}=A_{X} \cos 2 \phi+B_{X} \cos \phi+C_{X}+D_{X} \sin 2 \phi+E_{X} \sin \phi
$$

For the general tetrasubstituted case the equation takes the form

$$
\begin{aligned}
& { }^{3} J=\left(A+a \sum \Delta \chi_{i}\right) \cos 2 \phi+\left(B+b \sum \Delta \chi_{i}\right) \cos \phi+C+c \sum \Delta \chi_{i} \\
& +e \sum \psi \Delta \chi_{i} \sin 2 \phi+f \sum \psi \Delta \chi_{i} \sin \phi
\end{aligned}
$$

where $\psi$ is a parameter, taking the value -1 or +1 according to the relative position of the substituent X relative to the coupled protons. The definition of $\psi$ for each possible position is shown in Figure 1.

\section{INSERT FIGURE 1}

These effects were taken into account on the well-known Haasnoot-De LeeuwAltona (HLA) equation, ${ }^{[9]}$ which is by far the most commonly used one for the prediction of ${ }^{3} J_{\mathrm{Hн}}$ scalar couplings in organic compounds. Nonetheless, other equations were reported in the literature over the years. Some of them also included corrections for the H-C-C angle values as for instance the Barfield equation for the computation of ${ }^{3} J_{\mathrm{HH}}$ couplings for dihedral angles close to $180^{\circ}{ }^{[13]}$ Perhaps the most comprehensive empirical approach was that of Osawa ${ }^{[10]}$ which proposed a largely parametrized equation which takes into account those different structural parameters.

Since generally any coupling of the type X-A-B-Y can be fairly approximated to three-term Karplus Fourier series, over the years a large quantity of equations were reported in literature where nuclei different than ${ }^{13} \mathrm{C}$ or ${ }^{1} \mathrm{H}$ can appear at any of the four positions in the coupling path. This includes for instance ${ }^{3} J_{\mathrm{CH}}$ couplings, 
equations for fluorine and phosphorous systems, ${ }^{3} \mathrm{H}_{\mathrm{HH}}$ couplings in amine and alcohols and many others. Importantly, many equations were developed for the prediction of ${ }^{3} \mathrm{~J}$ couplings in peptidic/protein systems and virtually equations for each possible coupling involving either peptidic chain dihedrals or those involving side-chain atoms were also reported in the literature. These couplings can then be employed as experimental restraints for the determination of the 3D structure of proteins along with NOE and RDC data in restricted molecular dynamics runs. Particular references to many of these equations are listed in Table 1Error!

\section{Reference source not found..}

However, many of this published research and developed equations do not easily reach the final organic chemistry or biochemistry audience since, save for the most well-known equations as Karplus or HLA, most of them were not implemented in available computer programs or existing implementations can be considered abandonware. Karplus and HLA equations, as well as a few other Karplus relationships, can be found in pocket computer calculators, as the Sweet-J ${ }^{[14]}$ or MestRe- ${ }^{[15]}$ programs. These programs allow representation of the Karplus $J(\phi)$ curve as well as prediction of the coupling for particular dihedral angles. In the case of the HLA equation, which depends on the electronegativity of $\alpha$-substituents, the nature of these is manually fed into the program via the graphical interface. Clearly, a most practical approach for organic molecules is to load those molecular geometries obtained from molecular modeling techniques into a 3D visualization environment and allow the user to select the atoms of interest. This approach has been implemented in popular modeling programs such as for instance PCModel ${ }^{[16]}$ or Maestro, ${ }^{[17]}$ but these implementations are limited to few equations. Some molecular dynamics programs as for instance, the well-known XPLOR-NIH program $^{[18]}$ allow the use of generic three-term Karplus equations with custom $A, B, C$ parameters, since most of the equations developed for peptide systems follow the simple three-term Fourier cosine series functional form.

Following our purpose to bring state of the art structural elucidation techniques to the organic chemist toolbox, ${ }^{[19]}$ we felt the necessity to provide a software which could compute a large variety of $J$-couplings directly from from 3D molecular files. It was the purpose of the program to go beyond the ${ }^{3} J_{\mathrm{HH}}$ couplings-Karplus/HLA 
equations analysis and facilitate the use of other types of scalar couplings, such as, for instance, $3_{\mathrm{CH}}$ couplings, coupling in heteroatomic systems, etc. In addition, the development of a modular software, with a completely documented programming interface would allow the research groups involved in equation development an easy way to implement, test and facilitate the general use of their developed equations. Desirable characteristic for the software should be:

- Parsering. Most popular 3D chemical formats should be read including direct reading of molecular-modeling (force-field and $a b$-initio) file formats.

- Graphical interface. A proper GUI for the control of computation and graphical analysis of the results should be available to the user.

- Multiplatform. Since different laboratories may use different operating systems the use of multiplatform development frameworks seemed to us mandatory.

- Chemical intelligence. Since empirical equations are restricted to particular types of couplings, the software should attempt to interpret bonding patterns to find which coupled pairs are computable with a particular equation.

Following the above guidelines we developed a module for the MSpin software system (called MSpin-JCoupling from now on) for the implementation of Karpluslike relationships and their application to the structural analysis of small organic molecules.

\section{Results and Discussion}

\section{Implementation}

The software has been entirely written in the $\mathrm{C}++$ language. The graphical interface was built using the multiplatform $Q t 5^{[20]}$ framework as well as the Qwt6 $^{[21]}$ package for some additional technical widgets and representation of Karplus curves. The program has been built on top of the MagNes C++ API, ${ }^{[22]}$ which provide chemical file parsering, molecular information handling procedures, as well as molecular OpenGL rendering. The MSpin-JCoupling module is currently 
distributed by the MestreLab Research company as a binary commercial version. Users can either deploy their equations inside the MSpin program using the deployed headers and libraries. The code is also available under the open-source GPL v2 license (with some additional freedoms for combined use with academic oriented software) on the MagNes project website along with documentation for the different $\mathrm{C}++$ classes. $^{[22]}$

\section{Description of the program}

\section{Loading of molecular geometries and conformational populations}

MSpin-JCoupling can read a wide variety of chemical 3D formats, including general formats as for instance XYZ, SDF or PDB files from popular molecular modeling programs (i.e., PCModel, Macromodel-Maestro, or Hyperchem) as well as ab-initio formats (Gaussian09, NwChem, Orca). To deal with the fact that ab initio computations involve a single file per conformation a set of conformers can be read at once by deploying the whole set of outputs into a single directory and using the MSpin "Open Directory" option. A sanity check is performed to verify that all conformers belong to the same molecule (note however that at the present stage the program only checks for chemical constitution but not for relative configuration). The program can compute conformationally average J-coupling values as

$$
J=\sum_{i} w_{i} J_{i}
$$

where $\underline{w}_{\mathrm{i}}$ are the conformational weights and $J_{\mathrm{i}}$ are the values computed for a particular conformation. The conformational weights can: (i) be determined by reading the potential energies from the molecular files and converting them to populations at a given temperature using the Boltzmann equation or (ii) be just directly input by the user through the "Conformer" window. In this widget the user can either input directly the relative populations, which will then be normalized by the program, or the relative energies in $\mathrm{kcal} / \mathrm{mol}$. Boltzmann populations can be then computed by selecting an NVT ensemble and a given temperature. 


\section{Choosing equations and parameters}

The different equations currently available in MSpin-JCoupling are grouped into a box in the graphical interface of the program. They are grouped according to the type of coupling they provide. Left-clicking into an equation name will select it for computation while a right-click will display a box containing basic information about the equation, as well as a DOI hyperlink, when available, to the original reference. We strongly recommend the users to read the original literature to verify the scope and limitations of each particular equation.

In many cases, different reported equations for a given spin system $\mathrm{X}-\mathrm{A}-\mathrm{B}-\mathrm{Y}$ are just variations of empirically determined parameters, coefficients of a Fourier series in most of cases, for a same functional form. In MSpin-JCoupling each equation can contain several sets of parameters. These different sets are displayed in the application widget. The user can choose the desired set as well as to modify them, store these modifications, and retrieve them from disk. It should be noted that in the case of the HLA equation, the authors provided in the original publication different sets of parameters that were indented to be employed for disubstitued, trisubstitued and tetrasubstitued cases. ${ }^{\left[{ }^{[9}\right.}$ In our implementation the program automatically recognizes the pattern from the chemical structure and automatically choose the proper set, regardless of the user's selection.

In Mspin-JCoupling we have deployed a rich collection of equations of general type. For the most commonly used coupling, the $3^{3} \mathrm{HH}_{\mathrm{H}}$, the most popular Karplus and HLA equations are provided, as well as a modified Díez-Donders equation, which uses atom rather than group-electronegativities. ${ }^{[23]}$ For the ${ }^{3} J_{\mathrm{CH}}$ couplings we provide a simple Karplus relationship, as well as the recently proposed equation by Bifulco and coworkers. ${ }^{[2]}$ This equation takes the functional form of the Altona equation but it was reparameterized for ${ }^{3} J_{\mathrm{CH}}$ couplings on the basis of DFT computations. Other implemented equations involve couplings to $\mathrm{NH}$ and $\mathrm{OH}$ protons, as well as fluorine and phosphorous system as well as specialized equations for peptide and glycoside systems. Note that the program is not necessarily restricted to vicinal ${ }^{3} \mathrm{XY}_{\mathrm{X}}$ couplings as illustrated by the presence of the ${ }^{2} J$ equation proposed by Carlomagno. ${ }^{[25]}$ The list of deployed equations along with references and the MSpin names is shown in Table 1 Error! Reference source not found.. 


\section{INSERT TABLE 1}

Once an equation is selected, the couplings to be computed can be picked by just selecting the coupled atoms in the provided OpenGL visor. Noteworthy, the system will not allow selecting atoms that cannot be part of the coupled pairs computable with the chosen equation. Note that the coupling pathway is determined using the breadth-first searching algorithm. ${ }^{[26]}$ For relatively large systems this can be a tedious task and the program offers a "Select all" option. By employing chemical intelligence, which must be implemented for each particular equation, all the computable couplings are selected and displayed on the screen. The "Show result" button will report the computed couplings for each particular conformation and the corresponding conformational average. This table can then be copied and directly pasted into a text document or a spreadsheet.

\section{Representation of Karplus curves}

Having a didactic purpose in mind and also as a practical tool for those groups working in the development of new equations, it is possible to represent Karplus curves for any selected $3 \mathrm{~J}$ equation using a specialized widget, The widget allows exporting of the graphics in most common raster formats, as well as vector the formats SVG or PDF. The user can choose the desired angle interval and the resolution of the curve. Additionally, a set of points can be exported and pasted into any spreadsheet.

\section{INSERT FIGURE 2}

\section{Running the program on command line mode}

The program offers the possibility to be run directly from the command line. It takes as input a structure file, or a directory containing files for several conformations, and a text input file with the indexes of the coupled atoms as well as the empirical equation and parameter sets to be used. Populations can be also specified in the input file. See the supporting information for an example of input and output formats.

\section{Implementation of equations}

New equations may share a common functional form with those already implemented in MSpin-JCouplings, such as in the case of most three-term Fourier 
series. Hence, they can be simply implemented by modifying the current set of parameters and saving them to disk. The parameters are serialized as a simple text file containing a list of name-value pairs.

\section{INSERT SCHEME 1}

\section{INSERT SCHEME 2}

However, if this mechanism is not sufficient, new equations can be implemented through an offered $\mathrm{C}++$ programming interface. The inheritance diagram, along with main classes brief description, is showed in Scheme 1. All implemented equations must inherit from the JEquation class. This base class possesses several pure virtual functions, which must be reimplemented. At the very least the user should override the GetJ method where the actual computation of the J-coupling must be placed. Each equation can contain several sets of parameters. Each particular parameter group, for instance $A, B$ and $C$ parameters is stored into an instance of the Parameter class as string/float pair values. The Parameter class offers overloading of stream operators in order to facilitate input of the parameters. Loading of parameters for the Altona equation implementation is illustrated in Scheme 2.

Additionally, there are other functions that the user may want to override to allow for better integration with MSpin. Some of these methods just facilitate retrieval of information about the equation such as the Name, CoupledNuclei, Group or Description methods. Other virtual methods should be overridden for recognition of bonding patterns. These are the SelectFirstAtom and SelectSecondAtom methods. If methods are properly overridden the MSpin engine to find all computable pairs for a given equation. For instance, in the case of the H-C-C-F the implementation of SelectFirstAtom will check if the selected atom is a hydrogen or fluorine attached to an $\mathrm{sp}^{3}$ carbon, whereas the SelectSecondAtom implementation verifies that a three-bond path involving the carbon atoms can be established between the two selected nuclei.

The programming interface also provides specialized classes, called HCCHEquation and CCCHEquation, for the implementation of H-C-C-H and C-C-C- 
H Karplus relationships. These classes already provide a SelectFirstAtom and SelectSecondAtom methods so they do not need to be implemented in particular classes.

Finaly, a template is provided for the development of new equations, which makes use of the qmake Qt5 tool ${ }^{[48]}$ for the building of the makefiles.

\section{Conclusions}

A new program, MSpin-JCoupling, has been designed and developed to help organic and bioorganic chemists in the structural elucidation of organic compounds through use of scalar couplings. The program implements a wide variety of Karplus-like equations for different structural motifs as well as a plugin interface for the development and deployment of new equations.

\section{Supporting information}

Snapshots of several features available in the graphical interface of the program and commented examples for the implementation of Karplus-type equations using the MSpin-JCoupling programming interface.

\section{Acknowledgements}

The author thanks Xunta de Galicia for a Parga Pondal research contract and funding (Consellería de Educación 2009/071) and Spanish Ministerio de Ciencia e Innovación for financial support (PET2008-074).

\section{References}

[1] C. Altona, in Encyclopedia of $N M R$ (Ed.: W.N. York), D. M. Grant and R. Morris, 1996, pp. 4909-4923.

[2] B. Coxon, in Advances in Carbohydrate Chemistry and Biochemistry Volume 62, Elsevier, 2009, pp. 17-82.

[3] K. Kamienska-Trela, in Nuclear Magnetic Resonance, Royal Society of Chemistry, Cambridge, 2015, pp. 170-234.

[4] D. Neuhaus, M. P. Williamson, The Nuclear Overhauser Effect in Structural and Conformational Analysis Wiley-VCH, Wiley-VCH, 2000.

[5] R. R. Gil, A. Navarro-Vázquez, in Modern NMR Approaches to the 
Structure Elucidation of Natural Products: Volume 2: Data Acquisition and Applications to Compound Classes

, Royal Society of Chemistry, Cambridge, 2016, pp. 1-38.

[6] K. R. Gustafson, S. T. S. Chan, Y. Liu, in Modern NMR Approaches to the Structure Elucidation of Natural Products: Volume 2: Data Acquisition and Applications to Compound Classes

, Royal Society of Chemistry, Cambridge, 2016, pp. 39-70.

[7] M. Karplus, J. Chem. Phys. 1959, 30, 11-15.

[8] M. Karplus, J. Am. Chem. Soc. 1963, 85, 2870-2871.

[9] C. A. G. Haasnoot, F. A. A. M. de Leeuw, C. Altona, Tetrahedron 1980, 36, 2783-2792.

[10] K. Imai, E. Ōsawa, Magn. Reson. Chem. 1990, 28, 668-674.

[11] P. L. Durette, D. Horton, Magn. Reson. Chem. 1971, 3, 417-427.

[12] K. G. R. Pachler, Tetrahedron 1971, 27, 187-199.

[13] W. B. Smith, M. Barfield, Magn. Reson. Chem. 1993, 31, 696-697.

[14] G. Balacco, J. Chem. Inf. Comput. Sci. 1996, 36, 885-887.

[15] A. Navarro-Vázquez, J. C. Cobas, F. J. Sardina, J. Casanueva, E. Díez, J. Chem. Inf. Comput. Sci. 2004, 44, 1680-1685.

[16] K. E. Gilbert, PCModel 10.0, Serena Software

[17] Schrödinger Release 2017-2: MS Jaguar, Schrödinger, LLC, New York, NY, 2017.

[18] C. D. Schwieters, J. J. Kuszewski, N. Tjandra, G. Marius Clore, J. Magn. Reson. 2003, 160, 65-73.

[19] A. Navarro-Vázquez, Magn. Reson. Chem. 2012, 50, S73-S79.

[20] http://www.qt.io

[21] http://qwt.sourceforge.net

[22] http://magnes.sourceforge.io

[23] E. Díez, J. San-Fabián, J. Guilleme, C. Altona, L. A. Donders, Mol. Phys. 2006, 68, 49-63.

[24] G. Palermo, R. Riccio, G. Bifulco J. Org. Chem. 2010, 75, 19821991.

[25] J. Fohrer, U. Reinscheid, M. Hennig, T. Carlomagno, Angew. Chem. Int. Ed. 2006, 45, 7033-7036.

[26] S. S. Skiena, in The Algorithm Design Manual, Springer London, London, 2012, pp. 103-144.

[27] R. Aydin, H. Günther, Magn. Reson. Chem. 1990, 28, 448-457.

[28] H. Fukui, T. Baba, H. Inomata, K. Miura, H. Matsuda, Mol. Phys. 1997, 92, 161-165.

[29] H. Fukui, T. Baba, Y. Kurogi, J. Chem. Phys. 2000, 112, 3532-3539.

[30] I. Alkorta, J. Elguero, Theor. Chem Acc. 2004, 111, 31-35.

[31] H. Zhao, Q. Pan, W. Zhang, I. Carmichael, A. S. Serianni, J. Org. Chem. 2007, 72, 7071-7082.

[32] G. Govil, Mol. Phys.1971, 21, 953-957.

[33] C. Thibaudeau, J. Plavec, J. Chattopadhyaya, 1998, 63, 4967-4984.

[34] C. Rabiller, A. Dehnel, G. Lavielle, Can. J. Chem. 1982, 60, 926933.

[35] J. Thiem, B. Meyer, Org. Magn. Reson. 1978, 11, 50-51.

[36] L. F. Kao, M. Barfield, J. Am. Chem. Soc. 1985, 107, 2323-2330. 
[37] P. Mohanakrishnan, K. R. K. Easwaran, Biopolymers 1979, 18, 1769-1774.

[38] J.-S. Hu, A. Bax, J. Am. Chem. Soc. 1996, 118, 8170-8171.

[39] C. Pérez, F. Löhr, H. Rüterjans, J. M. Schmidt, J. Am. Chem. Soc. 2001, 123, 7081-7093.

[40] A. C. Wang, A. Bax, J. Am. Chem. Soc. 1995, 117, 1810-1813.

[41] J.-S. Hu, A. Bax, J. Am. Chem. Soc. 1997, 119, 6360-6368.

[42] M. T. Cung, G. Boussard, B. Vitoux, C. R. Acad. Sci. Serie C 1980, 291-294.

[43] C. F. Hammer, S. Chandrasegaran, J. Am. Chem. Soc. 1984, 106, 1543-1552.

[44] A. Pardi, M. Billeter, K. Wüthrich, J. Mol. Biol. 1984, 180, 741-751.

[45] M. J. Milton, R. Harris, M. A. Probert, R. A. Field, S. W. Homans, Glycobiology 1998, 8, 147-153.

[46] F. Cloran, I. Carmichael, A. S. Serianni, J. Am. Chem. Soc. 1999, 121, 9843-9851.

[47] I. Tvaroška, M. Hricovíni, E. Petráková, Carbohydr. Res. 1989, 189, 359-362.

[48] Qt5 qmake manual: http://doc.qt.io/qt-5/qmake-manual.html. 


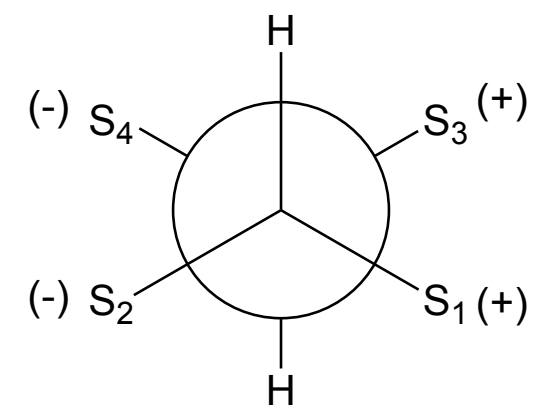

Figure 1. Sign of the parameter for each group in a tetrasubstitued ethane

Table 1. Implemented equations in the MSpin-JCoupling program

\begin{tabular}{|c|c|c|c|c|c|}
\hline Type & $\mathrm{X}$ & A & B & $\mathrm{Y}$ & Name \\
\hline \multicolumn{6}{|l|}{ General } \\
\hline \multicolumn{6}{|l|}{$3 \mathbf{J H C C H}$} \\
\hline $3 J_{\mathrm{HCCH}}$ & $\mathrm{H}$ & $\mathrm{C}_{\mathrm{sp} 3}$ & $\mathrm{C}_{\mathrm{sp} 3}$ & $\mathrm{H}$ & Karplus $^{[8]}$ \\
\hline $3 J_{\mathrm{HCCH}}$ & $\mathrm{H}$ & $\mathrm{C}_{\mathrm{sp} 3}$ & $\mathrm{C}_{\mathrm{sp} 3}$ & $\mathrm{H}$ & Altona $^{[9]}$ \\
\hline $3 \int_{\mathrm{HCCH}}$ & $\mathrm{H}$ & $\mathrm{C}_{\mathrm{sp} 3}$ & $\mathrm{C}_{\mathrm{sp} 3}$ & $\mathrm{H}$ & Diez-Donders $^{[1]}$ \\
\hline $3 J_{\mathrm{CCCH}}$ & $\mathrm{C}_{\mathrm{sp} 3}$ & $\mathrm{C}_{\mathrm{sp} 3}$ & $\mathrm{C}_{\mathrm{sp} 3}$ & $\mathrm{H}$ & Kalinowsky-[C-C-C-H] ${ }^{[27]}$ \\
\hline$\sqrt[3]{\mathrm{CCCH}}$ & $\mathrm{C}_{\mathrm{sp} 3}$ & $\mathrm{C}_{\mathrm{sp} 3}$ & $\mathrm{C}_{\mathrm{sp} 3}$ & $\mathrm{H}$ & Bifulco-[C-C-C-H] ${ }^{[24]}$ \\
\hline 3J $\mathrm{HCNH}$ & $\mathrm{H}$ & $\mathrm{C}_{\mathrm{sp} 3}$ & $\mathrm{~N}_{\mathrm{sp} 3}$ & $\mathrm{H}$ & General-[H-C-N-H] ${ }^{[28-30]}$ \\
\hline$\sqrt[3]{\text { нсон }}$ & $\mathrm{H}$ & $\mathrm{C}_{\mathrm{sp} 3}$ & 0 & $\mathrm{H}$ & General-[H-C-O-H] ${ }^{[28,30,31]}$ \\
\hline$\sqrt[3]{\text { нССF }}$ & $\mathrm{H}$ & $\mathrm{C}_{\mathrm{sp} 3}$ & $\mathrm{C}_{\mathrm{sp} 3}$ & $\mathrm{~F}$ & Govil-[H-C-C-F] $^{[32,33]}$ \\
\hline $3 / \mathrm{CNCP}$ & $\mathrm{C}$ & $\mathrm{N}$ & $\mathrm{C}$ & $\mathrm{P}(\mathrm{O}) \mathrm{OR}_{3}$ & Rabiller-[C-N-C-P] ${ }^{[34]}$ \\
\hline$\sqrt[3]{3}$ сссР & $\mathrm{C}$ & $\mathrm{C}$ & $\mathrm{C}$ & $\mathrm{P}(0) \mathrm{OR}_{3}$ & Thiem-[C-C-C-P] ${ }^{[35]}$ \\
\hline \multicolumn{6}{|l|}{ Peptides } \\
\hline \multicolumn{6}{|l|}{$3 J_{\mathrm{cc}}$} \\
\hline${ }^{3} J_{\mathrm{C}(=0), \mathrm{N}, \mathrm{C}_{\alpha}, \mathrm{C}_{\beta}}$ & $\mathrm{C}(=0)$ & $\mathrm{N}$ & $\mathrm{C}_{\alpha}$ & $\mathrm{C}_{\beta}$ & Barfield-[C(O)-N-Ca-Cb] \\
\hline
\end{tabular}




\begin{tabular}{|c|c|c|c|c|c|}
\hline & & & & & {$[36,37]$} \\
\hline${ }^{3} J_{\mathrm{C}(=0), \mathrm{N}, \mathrm{C}}, \mathrm{C}(=0)$ & $C(=0)$ & $\mathrm{N}$ & $\mathrm{C}_{\alpha}$ & $C(=0)$ & Bax-[C(O)-N-Ca-C(O)] $]^{[38]}$ \\
\hline${ }^{3} J_{C(=0), C_{\alpha}, C_{\beta} C g}$ & $C(=0)$ & $\mathrm{C}_{\alpha}$ & $\mathrm{C}_{\beta}$ & $\mathrm{Cg}$ & Schmidt- $[\mathrm{C}(\mathrm{O})-\mathrm{Ca}-\mathrm{Cb}-\mathrm{Cg}]^{[39]}$ \\
\hline \multicolumn{6}{|l|}{$3 J_{\mathrm{CH}}$} \\
\hline $3 J_{\mathrm{C}(=0), \mathrm{N}, \mathrm{C} \alpha, \mathrm{H}}$ & $\mathrm{C}=(0)$ & $\mathrm{N}$ & $\mathrm{C}_{\alpha}$ & $\mathrm{H}$ & Barfield-[C(O)-N-Ca-H] \\
\hline$\sqrt[3]{\mathrm{C}(=0), \mathrm{N}, \mathrm{C} \alpha, \mathrm{H}}$ & $C(=0)$ & $\mathrm{N}$ & $\mathrm{C}_{\alpha}$ & $\mathrm{H}$ & $\mathrm{Bax}[\mathrm{C}(\mathrm{O})-\mathrm{N}-\mathrm{Ca}-\mathrm{H}]^{[40]}$ \\
\hline${ }^{3} J_{\subset \beta, C \alpha, N, H}$ & $\mathrm{C}_{\beta}$ & $\mathrm{C}_{\alpha}$ & $\mathrm{N}$ & $\mathrm{H}$ & $\mathrm{Bax}-[\mathrm{Cb}-\mathrm{Ca}-\mathrm{N}-\mathrm{H}]^{[41]}$ \\
\hline $3_{\mathrm{CH} 3, \mathrm{~N}, \mathrm{C} \alpha, \mathrm{H}}$ & $\mathrm{C}\left(\mathrm{H}_{3}\right)$ & $\mathrm{N}$ & $\mathrm{C}_{\alpha}$ & $\mathrm{H}$ & Cung-[CH3-N-Ca-H] $]^{[42]}$ \\
\hline $3 J_{C=0, C \alpha,}, C \beta, H$ & $C(=0)$ & $\mathrm{C}_{\alpha}$ & $\mathrm{C}_{\beta}$ & $\mathrm{H}$ & Schmidt-[C(O)-Ca-Cb-H] $]^{[39]}$ \\
\hline${ }^{3} J_{C \gamma, C}, \mathrm{C} \alpha, \mathrm{N}, \mathrm{H}$ & $\mathrm{C} \gamma$ & $\mathrm{C}_{\beta}$ & $\mathrm{C}_{\alpha}$ & $\mathrm{H}$ & Schmidt-[H-Ca-Cb-Cg] ${ }^{[39]}$ \\
\hline \multicolumn{6}{|l|}{$3 J_{\mathrm{NH}}$} \\
\hline $3 J_{\mathrm{H}, \mathrm{C}}, \mathrm{C}(=\mathrm{O}), \mathrm{N}$ & $\mathrm{H}$ & $\mathrm{C}_{\alpha}$ & $C(=0)$ & $\mathrm{N}$ & Bax-[H-Ca-C(O)-N] $]^{[40]}$ \\
\hline $3 J_{N, C \alpha, C \beta, H}$ & $\mathrm{~N}$ & $\mathrm{C}_{\alpha}$ & $\mathrm{C}_{\beta}$ & $\mathrm{H}$ & Schmidt-[N-Ca-Cb-H $]^{[39]}$ \\
\hline \multicolumn{6}{|l|}{$3_{\mathrm{FH}}$} \\
\hline $3_{F, N, C \alpha, H}$ & $\mathrm{~F}$ & $\mathrm{~N}$ & $\mathrm{C}_{\alpha}$ & $\mathrm{H}$ & Hammer-[F-N-Ca-H] $]^{[43]}$ \\
\hline \multicolumn{6}{|l|}{$3 J_{\mathrm{HH}}$} \\
\hline $3 J_{\mathrm{H} \mathrm{N}, \mathrm{C} \alpha, \mathrm{H}}$ & $\mathrm{H}$ & $\mathrm{N}$ & $\mathrm{C}_{\alpha}$ & $\mathrm{H}$ & Pardi-[H-N-Ca-H] $]^{[44]}$ \\
\hline $3 J_{\mathrm{H} \mathrm{C} \alpha, \mathrm{C}_{\beta}, \mathrm{H}}$ & $\mathrm{H}$ & $\mathrm{C}_{\alpha}$ & $\mathrm{C}_{\beta}$ & $\mathrm{H}$ & Schmidt-[H-Ca-Cb-H $]^{[39]}$ \\
\hline \multicolumn{6}{|l|}{$3 J_{\mathrm{CN}}$} \\
\hline & $\mathrm{N}$ & $\mathrm{C}_{\alpha}$ & $\mathrm{C}_{\beta}$ & $\mathrm{C} \gamma$ & Schmidt-[N-Ca-Cb-Cg] $]^{[39]}$ \\
\hline \multicolumn{6}{|l|}{ Glycosides } \\
\hline${ }^{2} J_{\mathrm{C}, \mathrm{O}, \mathrm{H}}$ & $\mathrm{C}$ & 0 & --- & $\mathrm{H}$ & Carlomagno-[C-O-H] $]^{[25]}$ \\
\hline${ }^{3} J_{C, O, C, C}$ & $\mathrm{C}$ & 0 & $\mathrm{C}$ & $\mathrm{C}$ & Milton- $[\mathrm{C}-\mathrm{O}-\mathrm{C}-\mathrm{C}]^{[45]}$ \\
\hline $3 J_{\mathrm{C}, \mathrm{C}, \mathrm{O}, \mathrm{H}}$ & $\mathrm{C}$ & $\mathrm{C}$ & 0 & $\mathrm{H}$ & Serianni-[C1-C2-O-H] ${ }^{[31]}$ \\
\hline$\sqrt[3]{\mathrm{C}, \mathrm{O}, \mathrm{C}, \mathrm{H}}$ & $\mathrm{C}$ & 0 & $\mathrm{C}$ & $\mathrm{H}$ & Serianni-[C-O-C-H] $]^{[46]}$ \\
\hline $3_{\mathrm{C}, \mathrm{O}, \mathrm{C}, \mathrm{H}}$ & $\mathrm{C}$ & 0 & $\mathrm{C}$ & $\mathrm{H}$ & Tvaroska-[C-O-C-H] $]^{[47]}$ \\
\hline
\end{tabular}

a) Phosphonate group 


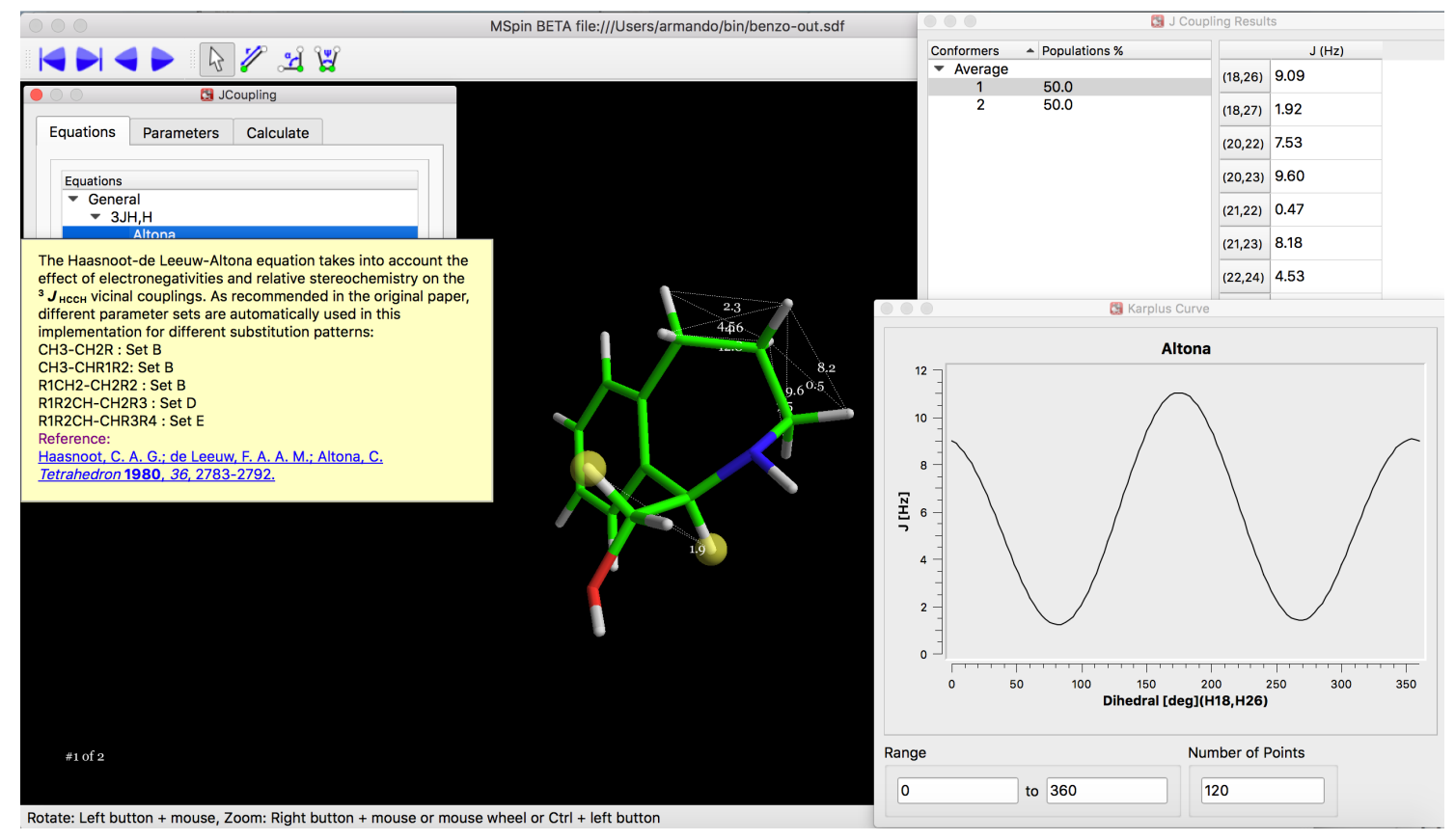

Figure 2. General screenshot for the MSpin-JCoupling application

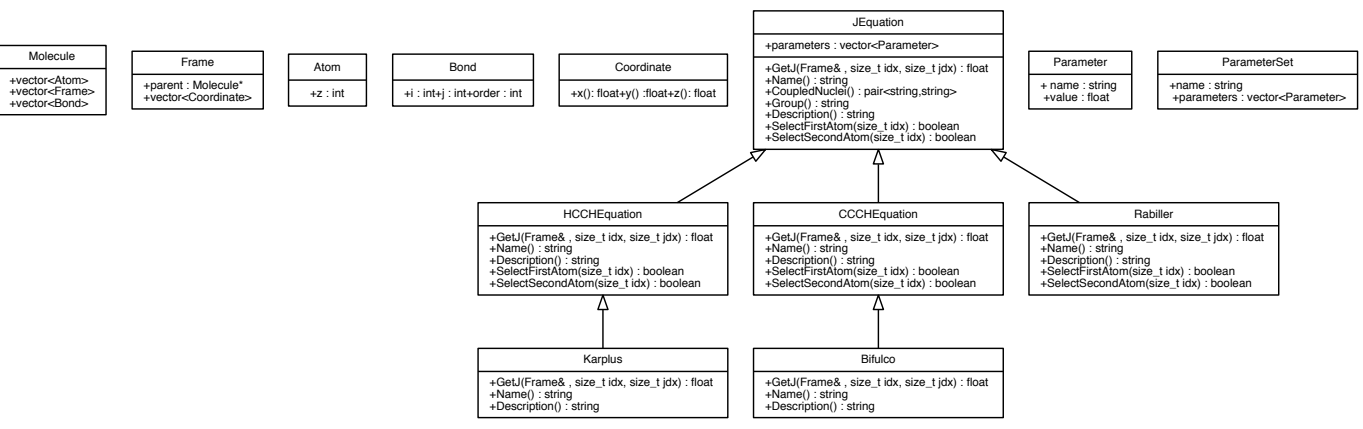

Scheme 1. Main classes and inheritance diagram for MSpin-JCoupling 


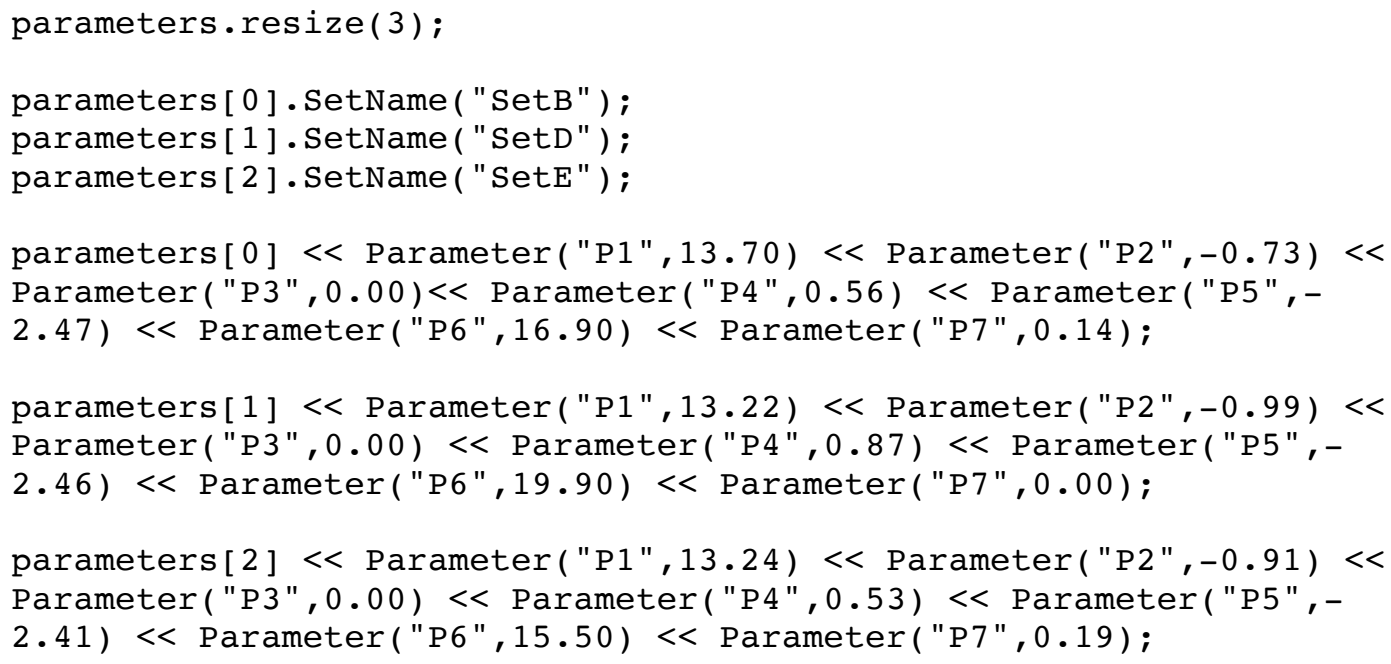

Scheme 2. Handling of parameter sets in MSpin-JCoupling 


\title{
MSpin-JCoupling. A modular program for J-coupling prediction and fast implementation of Karplus relationships
}

\author{
Armando Navarro-Vázquez
}

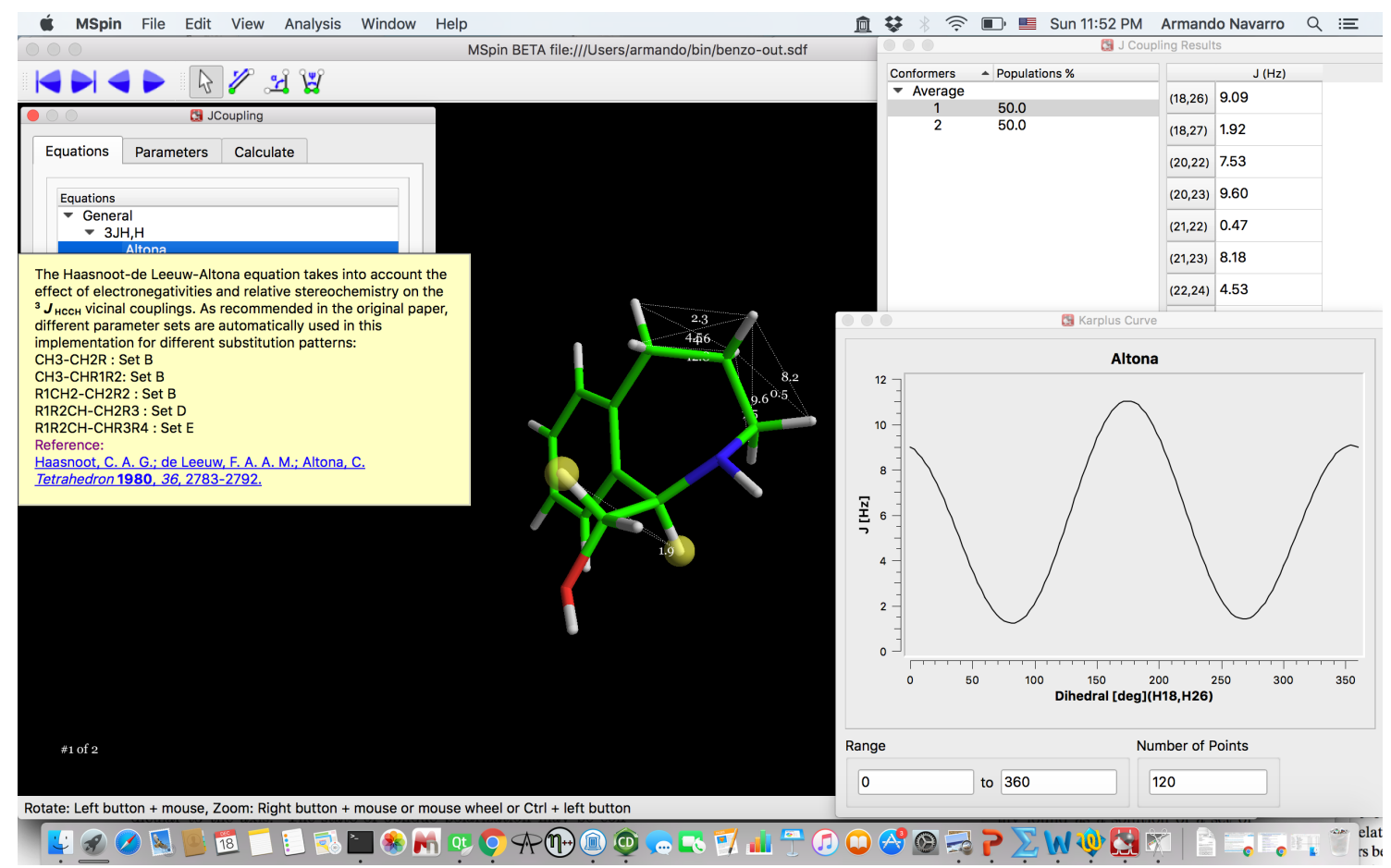

MSpin-JCoupling is a modular program for the prediction of scalar couplings using a large variety of Karplus relationships. The program was specially designed for small molecule analysis and can be run in graphical or command-line mode. The architecture of the program is highly modular and new equations can be rapidly implemented, through a complete $\mathrm{C}++$ programming interface, and deployed as run-time loadable plugins. 